\title{
Association between NF-кB expression and drug resistance of liver cancer
}

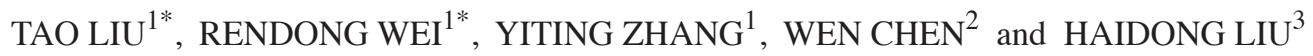 \\ ${ }^{1}$ Department of Hepatology, The Sixth People's Hospital of Qingdao, Qingdao, Shandong 266033; \\ ${ }^{2}$ Department of Infectious Diseases, The Affiliated Hospital of Southwest Medical University, Luzhou, Sichuan 646000; \\ ${ }^{3}$ Department of Digestive Diseases, The Sixth People's Hospital of Qingdao, Qingdao, Shandong 266033, P.R. China
}

Received December 28, 2017; Accepted September 11, 2018

DOI: $10.3892 / \mathrm{ol} .2018 .9640$

\begin{abstract}
Association between the expression of nuclear factor $\kappa \mathrm{B}(\mathrm{NF}-\kappa \mathrm{B})$ and the drug resistance of hepatoma cells was investigated. HepG-2 cells and HepG2/ADM cells were cultured, respectively. The morphology and status of the two groups of cells were observed by cell white light. The immunofluorescence by NF- $\mathrm{NB}$ and MDR1 staining on HepG-2 cells and HepG2/ADM cells, respectively, was applied and the fluorescence expression in the two groups of cells was observed. RT-qPCR was used to detect the expression of NF- $\kappa \mathrm{B}$ and MDR1 mRNA, the NF- $\kappa \mathrm{B}$ and MDR1 protein expression was detected by western blot analysis. The results of cell white illumination showed that the structure of HepG-2 and HepG2/ADM cells was complete and the cell morphology was normal, and there was no significant difference, and could be used for comparative study. Immunofluorescence staining showed that the expression of $\mathrm{NF}-\kappa \mathrm{B}$ and MDR1 in HepG-2 cells was very low, while the expression of $N F-\kappa B$ and MDR1 in HepG2/ADM cells was increased significantly. The RT-qPCR results showed that NF- $\mathrm{B}$ and MDR1 mRNA expression in HepG-2 cells was very low, while NF- $\mathrm{B}$ and MDR1 mRNA expression in HepG-2/ADM cells was significantly increased, and western blot results showed that $\mathrm{NF}-\kappa \mathrm{B}$ and MDR1 protein expression in HepG-2 cells was very low, while NF- $\kappa \mathrm{B}$ and MDR1 protein expression in HepG-2/ADM cells was increased significantly. The results of variance analysis showed that there was significant difference in the expression of the control group and paeonol group $(\mathrm{P}<0.01)$. In conclusion, the expression of NF- $\mathrm{BB}$ in the drug-resistant cells of liver cancer is closely related to the resistance-related gene $M D R 1$. This result may provide a new solution for the drug resistance of liver cancer.
\end{abstract}

Correspondence to: Dr Haidong Liu, Department of Digestive Diseases, The Sixth People's Hospital of Qingdao, 9 Fushun Road, Shibei, Qingdao, Shandong 266033, P.R. China

E-mail: qdlhd123@163.com

*Contributed equally

Key words: NF-кB, liver cancer, drug resistance, MDR1

\section{Introduction}

Liver cancer is a hepatic malignant tumor, which seriously endangers health. As its morbidity is on the increase annually, liver cancer has become a difficult problem to solve (1). In recent years, the therapeutic effect of liver cancer has been greatly improved along with the improvement in treatment methods and the application of numerous drugs (2). However, there is still no effective way to cure liver cancer due to multidrug resistance thereof.

Multidrug resistance refers to the resistance of tumor cells to various antitumor drugs (3). The molecular mechanism of tumor cell multidrug resistance is very complex. Therefore, in-depth research to solve this problem is imperative (4). P-glycoprotein (P-gp), the expression product of multidrug resistance gene $1(M D R 1)$, has ATP-dependent transmembrane transport activity, which can transport drugs to cells and induce drug resistance (5). Nuclear factor- $\kappa \mathrm{B}(\mathrm{NF}-\kappa \mathrm{B})$, participates in information transmission in defense response, tissue damage and stress, cell differentiation, apoptosis, and tumor growth inhibition (6).

In the present study, the molecular mechanism of drug resistance in liver cancer was explored by establishing HepG-2 and HepG-2/ADM cell lines and applying immunofluorescence, reverse transcription-polymerase chain reaction (RT-qPCR) and western blot analysis to study the association between $\mathrm{NF}-\kappa \mathrm{B}$ expression and liver cancer resistance, in order to provide experimental evidence for the prevention and treatment of liver cancer.

\section{Materials and methods}

Cell lines. The HepG-2 and drug-resistant HepG2/ADM cell lines were purchased from the American Type Culture Collection (ATCC), and Guangzhou Dahui Biotechnology Co., Ltd. (Guangzhou, China).

Main reagents. Dulbecco's modified Eagle's medium (DMEM) (Gibco, Carlsbad, CA, USA); fetal bovine serum (FBS) (Gibco); trypsin (Gibco); phosphate-buffered saline (PBS) (HyClone, Logan, UT, USA) bicinchoninic acid (BCA) protein assay kit (Beyotime Co., Shanghai, China); TRIzol total RNA extraction kit (Tiangen Biotech Co., Ltd., Beijing, 
China); RT-PCR kit (Tiangen Biotech Co., Ltd.); rabbit antihuman GAPDH, NF- $\kappa \mathrm{B}$ and MDR1 monoclonal antibodies, goat anti-rabbit secondary HRP and fluorescence secondary polyclonal antibodies (cat. nos. 2118, 4764, 13342, 7074, 4412, respectively; Cell Signaling Technology, Inc.; Danvers, MA, USA). The study was approved by the Ethics Committee of The Sixth People's Hospital of Qingdao (Qingdao, China).

Cell culture. HepG-2 and HepG2/ADM cells were cultured in DMEM containing $10 \%$ FBS in a constant temperature incubator with $5 \% \mathrm{CO}_{2}$ at $37^{\circ} \mathrm{C}$. The culture medium was changed every 2 days. The cells were placed onto a 6 -well plate in good condition for white light images and immunofluorescence staining, and mRNA and protein were extracted, respectively, for RT-PCR and western blot analysis.

Immunofluorescence staining. HepG-2 and HepG2/ADM cells were, respectively, inoculated into a 6-well plate at a density of $1 \times 10^{5} / \mathrm{ml}$ with $1 \mathrm{ml}$ in each well. The cells were then cultured for $24 \mathrm{~h}$, at $37^{\circ} \mathrm{C}$ and collected. Cell culture fluid was taken, and the cells were washed by PBS, fixed by $10 \%$ formalin, sealed with $5 \%$ skim milk and incubated for $1 \mathrm{~h}$ at $37^{\circ} \mathrm{C}$, followed by the addition of NF- $\kappa \mathrm{B}$ and MDR1 primary antibodies (1:100) for incubation at $4^{\circ} \mathrm{C}$ overnight. The next day, the cells were cleaned with PBS 3 times, followed by the addition of fluorescent secondary antibody (1:500) for incubation at $37^{\circ} \mathrm{C}$ for 1 h. Fluorescence microscopy (Olympus Corporation; Tokyo, Japan) was used to observe the expression of protein.

$R T$ - $q P C R$. HepG-2 and HepG2/ADM cells were, respectively, inoculated into 6 -well plates at a density of $1 \times 10^{5} / \mathrm{ml}$ with $1 \mathrm{ml}$ in each well. After $24 \mathrm{~h}$, the two kinds of cellular RNA were extracted according to instructions of the RNAiso Plus kit. The cells were rapidly transferred into $1 \mathrm{ml}$ TRIzol reagent, and extracted according to the protocol of the RNAiso Plus kit, and centrifuged for $5 \mathrm{~min}$ at $12,000 \mathrm{xg}$, at $4^{\circ} \mathrm{C}$. The supernatant was carefully removed and added with $100 \mu \mathrm{l}$ chloroform, mixed evenly, placed for $5 \mathrm{~min}$ at room temperature, and centrifuged at $12,000 \mathrm{x} \mathrm{g}$ and $4^{\circ} \mathrm{C}$ for $5 \mathrm{~min}$. The supernatant was removed carefully. Then the supernatant was added with the same volume of isopropanol, placed at room temperature for $10 \mathrm{~min}$ and centrifuged at $12,000 \mathrm{x} \mathrm{g}$, at $4^{\circ} \mathrm{C}$ for $10 \mathrm{~min}$. The precipitation was washed with $75 \%$ ethanol. Finally, RNase-free water was added to completely dissolve it. The ratio of $\mathrm{OD}_{260} / \mathrm{OD}_{280}$ and the concentration of RNA were measured. The purity and content of the extracted RNA samples were calculated. Then the samples were sub-packaged and stored at $-80^{\circ} \mathrm{C}$ for subsequent experiment. According to the ratio of the PrimeScript ${ }^{\circledR}$ RT reagent kit with cDNA Eraser kit, the reverse transcriptase solution was prepared, and the RNA samples were added for reverse transcription to obtain cDNA. The level of mRNA was measured according to the SYBR ${ }^{\circledR}$ Premix Ex Taq $^{\mathrm{TM}}$ II (Tli RNase H Plus) kit. The primer sequences of the corresponding RNA are shown in Table I. The cycle threshold (Cq) value was analyzed using the $2-^{\Delta \Delta \mathrm{Cq}}$ method (7).

Western blot analysis. According to the manual of the total protein extraction kit, the cells were lysed and centrifuged at $4^{\circ} \mathrm{C}$ and $12,000 \times \mathrm{g}$ for $10 \mathrm{~min}$. The supernatant was collected as the total protein. The concentration of total protein was
Table I. Primer sequences of related genes in RT-qPCR.

\begin{tabular}{lc}
\hline Gene name & Primer sequence \\
\hline$N F-\kappa B$ & F: 5'-3' AGCACAGATACCACCAAGACC \\
& R: 3'-5' GGGCACGATTGTCAAAGAT \\
MDRl & F: 5'-3' CCCATCATTGCAATAGCAGG \\
& R: 3'-5' GTTCAAACTTCTGCTCCTGA \\
$\beta$-actin & F: 5'-3' GAGCCGGGAAATCGTGCGT \\
& R: 3'-5' GGAAGGAAGGCTGGAAGATG
\end{tabular}

measured by BCA protein assay kit, and the protein was stored at $-80^{\circ} \mathrm{C}$ for later use. Total protein extraction and $2 \mathrm{X}$ loading buffer $(100 \mu \mathrm{l}+4 \mu \mathrm{l} \beta$-mercaptoethanol) were mixed at a volume ratio of $1: 1$, treated with boiling water bath for $5 \mathrm{~min}$, naturally cooled and stored in refrigerator at $4^{\circ} \mathrm{C}$. The appropriate proportion of $15 \%$ SDS-PAGE separation gel was prepared according to the molecular weight of the target protein and frozen for approximately $1 \mathrm{~h}$. Then 5\% SDS-PAGE concentrate gel was prepared and frozen for approximately half an hour. After the electrophoretic buffer solution was added, the denatured protein sample was added into the loading well, and the total protein content in each well was kept the same according to a certain protein concentration.

Electrophoresis was performed under a constant pressure of $220 \mathrm{~V}$ until the bromophenol blue reached the bottom of the gel. According to the molecular weight of target protein, the gel, a layer of PVDF membrane and six layers of filter paper were cut according to the size of the gel. PVDF membrane and the filter paper were immersed into methanol for $10 \mathrm{sec}$ and transferred into the transfer buffer. Then the positive pole-three layers of filter paper-PVDF membrane-gel-three layers of filter paper-negative order were placed on the membrane transfer instrument. Edge alignment needs attention to prevent blistering. After the membrane transfer under constant pressure of $110 \mathrm{~V}$ for $2 \mathrm{~h}$, the membrane attached with protein was blocked using $5 \%$ milk for $3 \mathrm{~h}$, the closed membrane was washed with TTBS for $5 \mathrm{~min}$, and incubated with the corresponding proportion of the primary $\mathrm{NF}-\kappa \mathrm{B}$, MDR1 and GAPDH antibodies (1:500) at $4^{\circ} \mathrm{C}$ overnight. The PVDF membrane was washed with TTBS for $5 \mathrm{~min}$, and then incubated with the corresponding secondary goat antirabbit secondary HRP polyclonal antibody $(1: 1,000)$ at room temperature for $3 \mathrm{~h}$. The membrane was washed again with TTBS (3 times, $10 \mathrm{~min}$ each time). Gel imager was warmed up for $30 \mathrm{~min}$, the A, B reagents of ECL kit were evenly mixed at the 1:1 ratio volume and were dropwise added to the PVDF membrane, followed by color development in the dark for $1 \mathrm{~min}$. The excess liquid was dried with the filter and the membrane was placed into the gel imager, followed by photography under dynamic integration model and observation. Lab Works 4.6 professional image analysis software (Lab-works Architecture; Wellington, New Zealand) was used to analyze the image.

Statistical analysis. The experimental data were expressed by mean \pm standard deviation (mean $\pm \mathrm{SD}$ ), and the experimental 

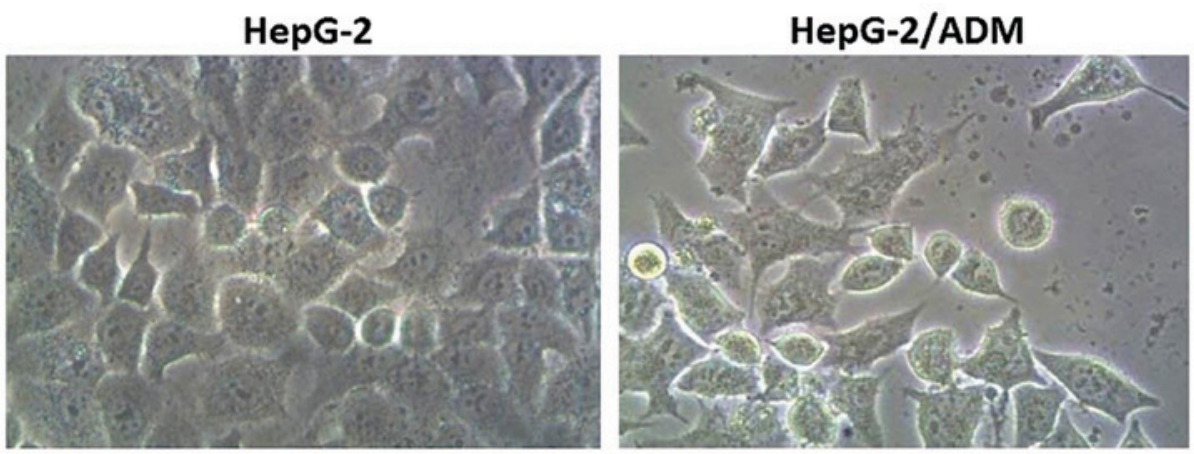

Figure 1. White images of HepG-2 and HepG2/ADM cells (x200). The structure of HepG-2 and HepG2/ADM cells is complete and the cell morphology is normal, and there are no significant differences.
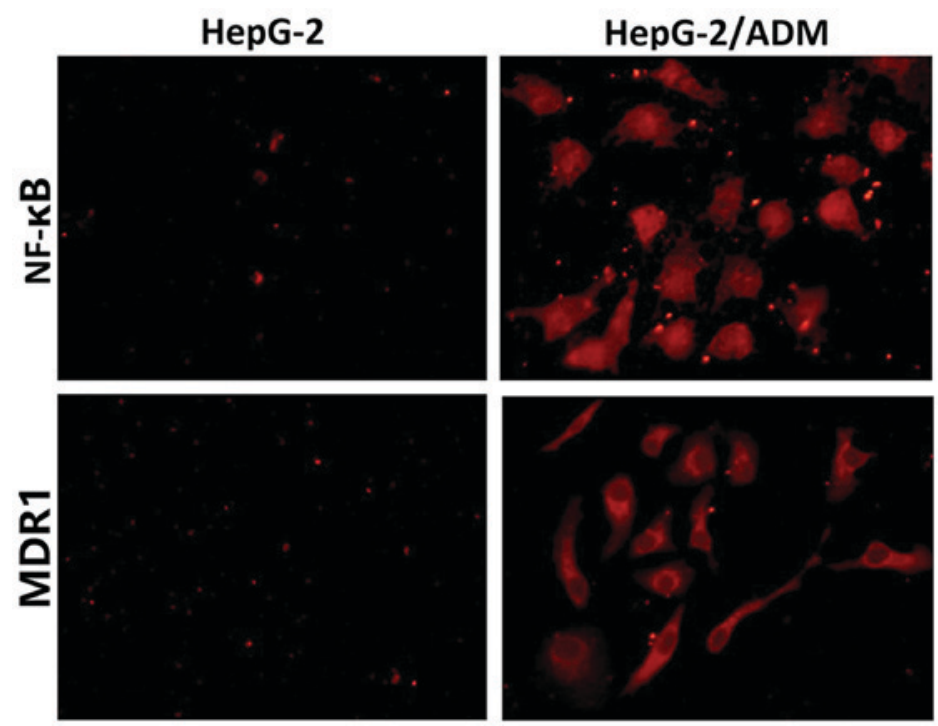

Figure 2. The results of immunofluorescence staining (x200) of HepG-2 and HepG2/ADM cells. Immunofluorescence staining shows that the expression levels of NF-kB and MDR1 in HepG-2 cells are very low, while those in HepG2/ADM cells are increased significantly.

results were analyzed by Statistical Product and Service Solutions (SPSS) 17.0 statistical software. The means between the two groups were compared by t-test. One-way ANOVA was used for the comparison of means among groups and the post hoc test was SNK test. P-test was used for pairwise comparison. $\mathrm{P}<0.05$ was considered to indicate a statistically significant difference.

\section{Results}

Cell white images. HepG-2 and HepG2/ADM cells were inoculated in 6-well plates, respectively. When the cells adhered well, cells in the two groups were observed via white images, and they were normal in morphology and could be used for the experiment. As shown in Fig. 1, the structures of HepG-2 and HepG2/ADM cells were complete and the cell morphology was normal, and there were no significant differences, and could be used for comparative study.

Results of immunofluorescence staining. NF- $\mathrm{B}$ and MDR1 immunofluorescence staining was performed for HepG-2 and HepG2/ADM cells, respectively (Fig. 2). Immunofluorescence staining showed that the expression levels of NF- $\kappa B$ and MDR1 in HepG-2 cells were very low, but those in HepG2/ADM cells were increased significantly. Therefore, the expression of $\mathrm{NF}-\kappa \mathrm{B}$ is closely related to the MDR1 of liver cancer cells.

Results of $R T-q P C R$. The total RNA was extracted from HepG-2 and HepG2/ADM cells. RT-qPCR showed that the expression levels of $\mathrm{NF}-\kappa \mathrm{B}$ and MDR1 mRNA in HepG-2 cells were very low, while those in HepG-2/ADM cells were increased significantly (Fig. 3).

Western blot results. Western blot analysis was performed for the protein extracted from HepG-2 and HepG2/ADM cell samples. As shown in Fig. 4, the expression levels of $\mathrm{NF}-\kappa \mathrm{B}$ and MDR1 protein in HepG-2 cells were very low, while those in HepG-2/ADM cells were increased significantly. It is concluded that the expression of $\mathrm{NF}-\kappa \mathrm{B}$ is closely related to the drug resistance of liver cancer, and related to the drug resistance associated protein MDR1.

\section{Discussion}

Liver cancer is the general name of two different malignant tumors, namely primary liver cancer and metastatic liver 

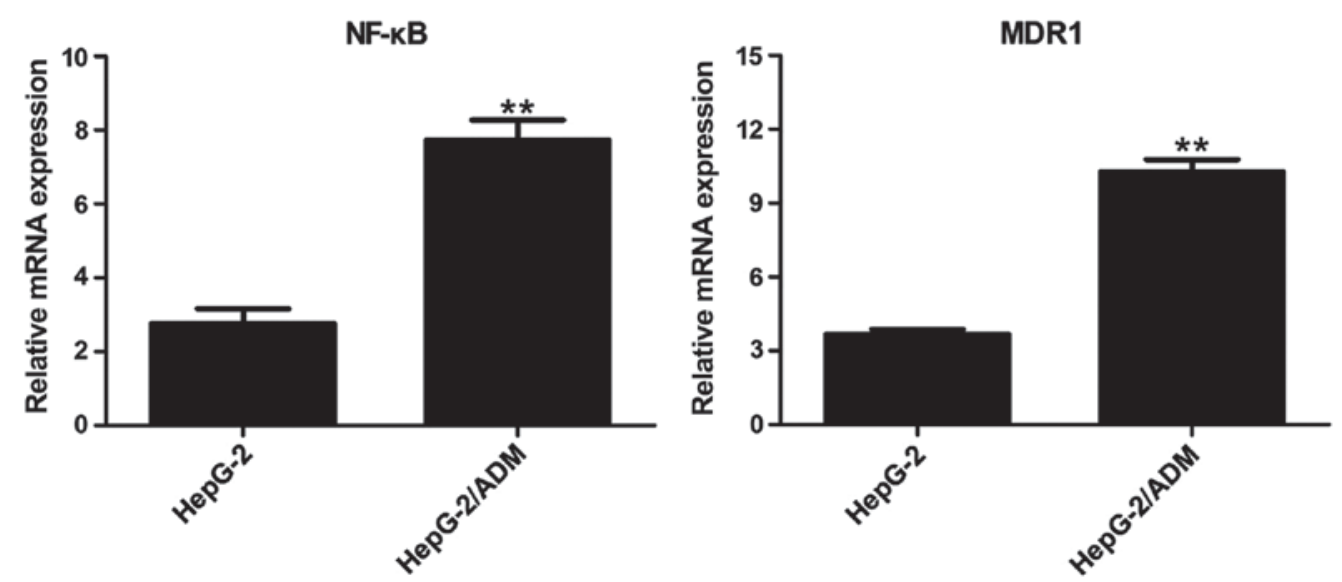

Figure 3. Expression levels of NF- $\mathrm{KB}$ and MDR1 mRNA in HepG-2 and HepG2/ADM cells. Compared with the HepG-2 cell group, ${ }^{* *} \mathrm{P}<0.01$ ( $\mathrm{n}=3$ ). The expression levels of NF- $\mathrm{KB}$ and MDR1 mRNA in HepG-2 cells are very low, while those in HepG-2/ADM cells are increased significantly.


Figure 4. Expression levels of NF- $\mathrm{KB}$ and MDR1 protein in HepG-2 and HepG2/ADM cells. Compared with the HepG-2 cell group, ${ }^{* *} \mathrm{P}<0.01$ ( $\mathrm{n}=3$ ). The expression levels of NF- $\mathrm{kB}$ and MDR1 protein in HepG-2 cells are very low, while those in HepG-2/ADM cells are increased significantly.

cancer, which seriously threatens people's health. The mortality rate of liver cancer is high around the world, and the number of male patients is increasing each year (8-11). In China, primary liver cancer has developed to be a major tumor second only to lung cancer (12). Liver cancer is a serious threat to life. At present, there are many therapeutic methods for liver cancer; however, no method can be used as complete cure, which is closely related to the multidrug resistance of liver cancer (13). In this study, the mechanism of drug resistance of liver cancer was studied through using liver cancer HepG-2 cells as the liver cancer model and the HepG-2/ADM cells as the drug resistance model of liver cancer.

The phenomenon of selecting cells against single cytotoxic drugs may develop cross resistance to a variety of drugs with different structures and cell targets is known as multiple resistance (14). MDR protein (MDRS) is a highly conserved ATP binding cassette transporter superfamily member. MDR is a top transmembrane protein and a component of the bloodbrain barrier function, which transports various drugs as a drug delivery pump from the brain to blood. The multidrug resistance gene is known to be ABCB1 on the human chromosome 7 (15).
The overexpression of drug transporter protein MDR1 is the main barrier in cancer chemotherapy. The role of MDR 1 in inducing cell apoptosis has been demonstrated (16) in several cell lines. MDR1 is an ATP-dependent pump to pump the negatively charged drug molecules out of the cell against a concentration gradient, resulting in the decrease of the intracellular drug concentration, and leading to the occurrence of drug resistance (17). Moreover, MDR1 can induce tumor drug resistance through reducing the concentration of drugs when reaching the target site by changing the $\mathrm{pH}$ value of cytoplasm and organelles, and directly participate in metastasis (18).

$\mathrm{NF}-\kappa \mathrm{B}$, as a nuclear transcription factor, regulates the expression of a large number of genes, which are critical for the regulation of cell apoptosis, viral replication, tumorigenesis, inflammation and various autoimmune diseases (19). NF- $\kappa \mathrm{B}$ can be activated by various stimuli, including growth factors, cytokines, lymphokine, ultraviolet, pharmaceutical activity and stress, which is considered to be a part of stress response. In its active form, $\mathrm{NF}-\kappa \mathrm{B}$ is isolated in the cytoplasm by the restraining of the I $\kappa$ B family members (20). Various stimuli are the main causes of the activation of phosphorylated $\mathrm{I} \kappa \mathrm{B}$ of $\mathrm{NF}-\kappa \mathrm{B}$, followed by 
its ubiquitination and subsequent degradation, resulting in the exposure of the nuclear location signal on the NF- $\kappa \mathrm{B}$ subunit and the subsequent transfer of the molecules to the nucleus (21).

In this study, HepG-2 cells and HepG2/ADM cells were cultured, respectively, as the model of liver cancer and liver cancer drug resistance. When the cells grew well in good conditions, they were placed onto 6-well plates. The morphology and status of two groups of cells were observed by cell white images. As a result, the structures of HepG-2 and HepG2/ADM cells were complete and the cell morphology was normal, and there were no significant differences. NF- $\kappa \mathrm{B}$ and MDR1 immunofluorescence staining were performed for HepG-2 and HepG2/ADM cells, respectively. The differences of NF- $\mathrm{B}$ and MDR1 fluorescence expression levels in two groups of cells were observed. The results of immunofluorescence staining showed that the expression levels of $N F-\kappa B$ and MDR1 were very low, while those in HepG2/ADM cells were increased significantly. The RT-qPCR results showed that the expression levels of NF- $\kappa \mathrm{B}$ and MDR1 mRNA in HepG-2 cells were very low, while those in HepG-2/ADM cells were significantly increased. At the same time, western blot results showed that NF- $\kappa \mathrm{B}$ and MDR1 protein expression levels in HepG-2 cells were very low, while those in HepG-2/ADM cells were increased significantly. Collectively, the expression of NF- $\kappa \mathrm{B}$ in the drug-resistant cells of liver cancer is closely related to the resistance related gene MDR1. This result may provide a new solution for the drug resistance of liver cancer.

\section{Acknowledgements}

Not applicable.

\section{Funding}

No funding was received.

\section{Availability of data and materials}

The datasets used and/or analyzed during the current study are available from the corresponding author on reasonable request.

\section{Authors' contributions}

TL and RW were responsible for PCR and western blot analysis. YZ and WC contributed to cell culture and immunofluorescence staining. HL and TL helped with statistical analysis. All authors read and approved the final manuscript.

\section{Ethics approval and consent to participate}

The study was approved by the Ethics Committee of The Sixth People's Hospital of Qingdao (Qingdao, China).

\section{Patient consent for publication}

Not applicable.

\section{Competing interests}

The authors declare that they have no competing interests.

\section{References}

1. Gottesman MM and Pastan I: Biochemistry of multidrug resistance mediated by the multidrug transporter. Annu Rev Biochem 62: 385-427, 1993.

2. Ambudkar SV, Dey S, Hrycyna CA, Ramachandra M, Pastan I and Gottesman MM: Biochemical, cellular, and pharmacological aspects of the multidrug transporter. Annu Rev Pharmacol Toxicol 39: 361-398, 1999.

3. Krishna $R$ and Mayer LD: Multidrug resistance (MDR) in cancer. Mechanisms, reversal using modulators of MDR and the role of MDR modulators in influencing the pharmacokinetics of anticancer drugs. Eur J Pharm Sci 11: 265-283, 2000.

4. Yusa K and Tsuruo T: Reversal mechanism of multidrug resistance by verapamil: Direct binding of verapamil to P-glycoprotein on specific sites and transport of verapamil outward across the plasma membrane of K562/ADM cells. Cancer Res 49: 5002-5006, 1989.

5. Loo TW and Clarke DM: Defining the drug-binding site in the human multidrug resistance P-glycoprotein using a methanethiosulfonate analog of verapamil, MTS-verapamil. J Biol Chem 276: 14972-14979, 2001.

6. Chin KV, Ueda K, Pastan I and Gottesman MM: Modulation of activity of the promoter of the human MDR1 gene by Ras and p53. Science 255: 459-462, 1992.

7. Livak KJ and Schmittgen TD: Analysis of relative geneexpression data using real time quantitative PCR and the 2(-Delta Delta C(T)) Method. Methods 25: 402-408, 2001.

8. Cornwell MM and Smith DE: SP1 activates the MDR1 promoter through one of two distinct G-rich regions that modulate promoter activity. J Biol Chem 268: 19505-19511, 1993.

9. Zhou G and Kuo MT: NF-kappaB-mediated induction of mdrlb expression by insulin in rat hepatoma cells. J Biol Chem 272: 15174-15183, 1997.

10. Fine RL, Chambers TC and Sachs CW: P-glycoprotein, multidrug resistance and protein kinase C. Stem Cells 14: 47-55, 1996.

11. Tang XY and Zhu YQ: Epigallocatechin-3-gallate suppressed the over-expression of HSP 70 and MDR1 induced by heat shock in SGC 7901. J Chemother 20: 355-360, 2008.

12. Chin KV, Tanaka S, Darlington G, Pastan I and Gottesman MM: Heat shock and arsenite increase expression of the multidrug resistance (MDR1) gene in human renal carcinoma cells. J Biol Chem 265: 221-226, 1990.

13. David GL, Yegnasubramanian S, Kumar A, Marchi VL, De Marzo AM, Lin X and Nelson WG: MDR1 promoter hypermethylation in MCF-7 human breast cancer cells: Changes in chromatin structure induced by treatment with 5-Aza-cytidine. Cancer Biol Ther 3: 540-548, 2004.

14. Yatouji S, El-Khoury V, Trentesaux C, Trussardi-Regnier A, Benabid R, Bontems F and Dufer J: Differential modulation of nuclear texture, histone acetylation, and MDR1 gene expression in human drug-sensitive and -resistant OV1 cell lines. Int J Oncol 30: 1003-1009, 2007.

15. Tang PM, Chan JY, Zhang DM, Au SW, Fong WP, Kong SK, Tsui SK, Waye MM, Mak TC and Fung KP: Pheophorbide a, an active component in Scutellaria barbata, reverses P-glycoproteinmediated multidrug resistance on a human hepatoma cell line R-HepG2. Cancer Biol Ther 6: 504-509, 2007.

16. Karin M and Lin A: NF-kappaB at the crossroads of life and death. Nat Immunol 3: 221-227, 2002.

17. Pahl HL: Activators and target genes of Rel/NF-kappaB transcription factors. Oncogene 18: 6853-6866, 1999.

18. Ueda K, Pastan I and Gottesman MM: Isolation and sequence of the promoter region of the human multidrug-resistance (P-glycoprotein) gene. J Biol Chem 262: 17432-17436, 1987.

19. Israël A: The IKK complex, a central regulator of NF-kappaB activation. Cold Spring Harb Perspect Biol 2: a000158, 2010.

20. Aydin C, Sanlioglu AD, Bisgin A, Yoldas B, Dertsiz L, Karacay B, Griffith TS and Sanlioglu S: NF- $\mathrm{BB}$ targeting by way of IKK inhibition sensitizes lung cancer cells to adenovirus delivery of TRAIL. BMC Cancer 10: 584, 2010.

21. Nakanishi $\mathrm{C}$ and Toi M: Nuclear factor-kappaB inhibitors as sensitizers to anticancer drugs. Nat Rev Cancer 5: 297-309, 2005. 